\title{
Slowly varying amplitude approximation appraised by transfer-matrix approach
}

\author{
Zhi-Yuan Li \\ Institute of Physics, Chinese Academy of Sciences, P.O. Box 603, Beijing 100080, China \\ and Department of Physics, Hong Kong University of Science and Technology, Clear Water Bay, Kowloon, Hong Kong, China \\ Ben-Yuan Gu \\ CCAST (World Laboratory), P.O. Box 8730, Beijing 100080, China \\ Guo-Zhen Yang \\ Institute of Physics, Chinese Academy of Sciences, P.O. Box 603, Beijing 100080, China
}

(Received 7 June 1999)

\begin{abstract}
The slowly varying amplitude approximation that is widely adopted in nonlinear optics is appraised by the transfer-matrix method. Rigorous solution for second harmonic generation in nonlinear optical superlattices shows that this approximation is invalid when the reflection of the second harmonic (SH) wave from the crystal interface cannot be neglected. When the modulation period of the superlattice is comparable to the wavelength of the SH wave, the approximation is far from accurate even if no reflection from the crystal interface occurs. The transfer-matrix method may provide a novel approach to investigate various nonlinear optical processes in superlattices in a precise way. [S0163-1829(99)13139-4]
\end{abstract}

Since the invention of lasers, nonlinear optics has been developed into a fascinating field of broadest scope and influential proponents. ${ }^{1,2}$ Originated from experimental work by Franken and co-workers ${ }^{3}$ and theoretical work by Bloembergen and co-workers, ${ }^{4}$ this field now finds applications in many areas of sciences.

The propagation of nonlinear waves in nonlinear crystals is governed by the coupled-wave equations, which are based on Maxwell's equations. ${ }^{1}$ Understanding of fruitful nonlinear optical phenomena involves solving such complex nonlinear differential equations, a difficult task when one takes into account practical conditions in experiments. Thus in actual cases, several simplifying approximations are often made., Among them are the slowly varying amplitude (SVA) approximation, the infinite plane-wave approximation, and constant pump intensity approximation. From a realistic experimental view point, some corrections were made. For instance, sum-frequency generation with high conversion efficiency beyond constant pump intensity approximation ${ }^{1}$ and nonlinear processes excited by focused beams other than plane waves ${ }^{6}$ were investigated in detail. However, little attention was paid to the SVA approximation. Although it was revealed in Ref. 1 that the physical implication of this approximation consisted in neglecting the oppositely propagating nonlinear wave, this conclusion remains qualitative and obscure. Quantitative and clear appraisal of this approximation is necessary.

In this paper we will appraise the SVA approximation with use of the transfer matrix method. Our aim is to demonstrate the break down of the SVA approximation. Therefore, for concreteness, we focus our consideration on onedimensional (1D) nonlinear optical superlattices where the linear susceptibility is homogeneous, while second-order nonlinear susceptibility is modulated by alternate laminar domains with positive and negative polarizations. This can be accomplished in many ferroelectric crystals such as $\mathrm{LiNbO}_{3}$,
$\mathrm{LiTaO}_{3}$, and $\mathrm{KTiOPO}_{4}$ with patterned electrodes. The concept of Quasi-phase-matching ${ }^{4,7,8}$ has been realized in such periodic and quasi-periodic superlattices to achieve harmonic generations and other nonlinear processes with high efficiency. $^{8-18}$

The schematic configuration of superlattice is displayed in Fig. 1. The crystal (Region II) is assumed to lie inside a homogeneous background dielectric (Regions I and III) with a refractive index of $n_{b}$. The nonlinear crystal is excited with a plane wave of fundamental light $(\mathrm{FW})$, whose electric field reads $\mathrm{E}_{1}(z)=E e^{i k_{1} z}$ with $k_{1}=n_{1}(\omega) \omega / c$. Here $n_{1}(\omega)$ is the refractive index of crystal for the FW. The modulation function of nonlinear superlattice is a discrete function, where $\chi(z)=\chi_{i}$ in the $i$ th unit cell for $z_{i-1} \leqslant z \leqslant z_{i}$, and $\chi_{i}$ takes two discrete values, $d_{33}$ and $-d_{33}$ for the positive and negative polarization of unit cell, respectively. Assume that $z_{0}=0$ and $z_{n}=d$, where $d$ is the length of superlattice material.

In the approximation of negligible pump power depletion, the electric field $\mathrm{E}_{2}(z)$ of the second harmonic wave in the $1 \mathrm{D}$ superlattice satisfies the following inhomogeneous wave equation:

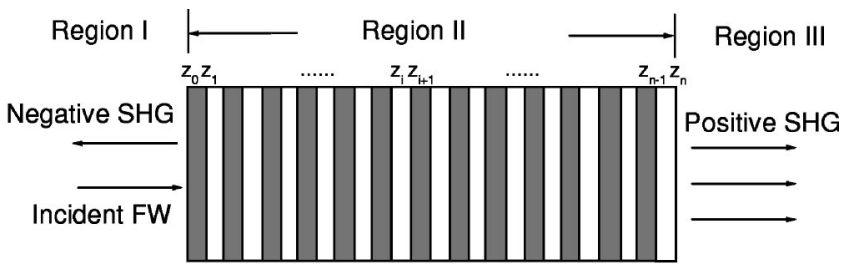

FIG. 1. Schematic configuration of 1D nonlinear optical superlattice (Region II) inside a homogeneous dielectric background (Regions I and III). Second-harmonic generation is excited by a plane fundamental light. The superlattice is composed of $n$ alternative laminar domains with positive (black cells) and negative (white cells) polarizations. 


$$
\nabla^{2} \mathrm{E}_{2}(z)+k_{2}^{2} \mathrm{E}_{2}(z)=-k_{0}^{2} \chi(z) \mathrm{E}_{1}^{2}(z),
$$

where $k_{0}=2 \omega / c$, and $k_{2}=n_{2}(2 \omega) k$ with $n_{2}$ being the refractive index for the SH wave. For most nonlinear crystals, $n_{1} \neq n_{2}$ due to the dispersion of light in crystals.

The solution of Eq. (1) in the $i$ th unit cell can be written as

$$
\mathrm{E}_{2}(z)=E_{i}^{+} e^{i k_{2} z}+E_{i}^{-} e^{-i k_{2} z}+E_{i} e^{i 2 k_{1} z}, \quad z_{i-1} \leqslant z \leqslant z_{i},
$$

where $E_{i}^{+}$and $E_{i}^{-}$are amplitudes of second harmonic (SH) waves propagating forwardly and backwardly, $E_{i}$ $=k_{0}^{2} \chi_{i} E^{2} /\left(4 k_{1}^{2}-k_{2}^{2}\right)$ is the amplitude of inhomogeneous $\mathrm{SH}$ wave. The transmitted and reflected SH waves in the dielectric background are both plane waves,

$$
\begin{gathered}
\mathrm{E}_{2}(z)=E_{t} e^{i k_{b} z}, \quad z>z_{n}=d, \\
\mathrm{E}_{2}(z)=E_{r} e^{-i k_{b} z}, \quad z<z_{0}=0,
\end{gathered}
$$

where $E_{t}$ and $E_{r}$ are both complex constants, and $k_{b}$ $=2 \omega n_{b} / c$. The intensity of second-harmonic generation (SHG) signal in the forward and backward directions are proportional to $I_{t}=n_{b}\left|E_{t}\right|^{2}$ and $I_{r}=n_{b}\left|E_{r}\right|^{2}$, respectively.

The amplitudes $\left\{E_{i}^{+}, E_{i}^{-}, i=1, \ldots, n\right\}$ as well as $E_{t}$ and $E_{r}$ can be solved through the match of boundary conditions at each interface of unit cell. At $z=z_{0}=0$ and $z=z_{n}=d$ we have

$$
S_{0} T_{1}=S_{r} T_{r}, \quad S_{n} T_{n}=S_{t} T_{t} .
$$

At the interface of $z=z_{i}$, we have the following recurrence formula:

$$
S_{i}\left(T_{i}-T_{i+1}\right)=\left(E_{i+1}-E_{i}\right) P_{i}, \quad i=1, \ldots, n-1 .
$$

The matrices in Eqs. (5) and (6) are defined as

$$
\begin{gathered}
S_{r}=\left(\begin{array}{cc}
e^{-i k_{b} z_{0}} & -e^{i 2 k_{1} z_{0}} \\
-k_{b} e^{-i k_{b} z_{0}} & -2 k_{1} e^{-i 2 k_{1} z_{0}}
\end{array}\right), \quad T_{r}=\left(\begin{array}{c}
E_{r} \\
E_{1}
\end{array}\right), \\
S_{t}=\left(\begin{array}{cc}
e^{i k_{b} z_{n}} & -e^{i 2 k_{1} z_{n}} \\
k_{b} e^{-i k_{b} z_{n}} & -2 k_{1} e^{-i 2 k_{1} z_{n}}
\end{array}\right), \quad T_{t}=\left(\begin{array}{c}
E_{t} \\
E_{n}
\end{array}\right),
\end{gathered}
$$

and

$$
\begin{gathered}
S_{i}=\left(\begin{array}{cc}
e^{i k_{2} z_{i}} & e^{-i k_{2} z_{i}} \\
k_{2} e^{i k_{2} z_{i}} & -k_{2} e^{-i k_{2} z_{i}}
\end{array}\right), \quad T_{i}=\left(\begin{array}{c}
E_{i}^{+} \\
E_{i}^{-}
\end{array}\right), \\
P_{i}=\left(\begin{array}{c}
e^{i 2 k_{1} z_{i}} \\
2 k_{1} e^{i 2 k_{1} z_{i}}
\end{array}\right) .
\end{gathered}
$$

From Eq. (6) we get

$$
T_{n}-T_{1}=-\sum_{i=1}^{n-1}\left(E_{i+1}-E_{i}\right) S_{i}^{-1} P_{i} .
$$

The substitution of Eq. (5) into Eq. (7) yields the amplitudes of the transmitted and reflected $\mathrm{SH}$ waves:

$$
E_{t}=a \sum_{i=1}^{n} E_{i}\left(e^{i \Delta k z_{i}}-e^{i \Delta k z_{i-1}}\right)+b \sum_{i=1}^{n} E_{i}\left(e^{i k_{3} z_{i}}-e^{i k_{3} z_{i-1}}\right),
$$

$$
E_{r}=s_{1} \sum_{i=1}^{n} E_{i}\left(e^{i \Delta k z_{i}}-e^{i \Delta k z_{i-1}}\right)+s_{2} \sum_{i=1}^{n} E_{i}\left(e^{i k_{3} z_{i}}-e^{i k_{3} z_{i-1}}\right) .
$$

The coefficients are

$$
\begin{gathered}
a=-\left(\frac{1}{2}+\frac{k_{1}}{k_{2}}\right)\left(\frac{1}{2}+\frac{k_{b}}{2 k_{2}}\right) / s s, \\
b=\left(\frac{1}{2}-\frac{k_{1}}{k_{2}}\right)\left(\frac{1}{2}-\frac{k_{b}}{2 k_{2}}\right) / s s, \\
s_{1}=-\left(\frac{1}{2}+\frac{k_{1}}{k_{2}}\right)\left(\frac{1}{2}-\frac{k_{b}}{2 k_{2}}\right) e^{i k_{5} d / s s,} \\
s_{2}=\left(\frac{1}{2}-\frac{k_{1}}{k_{2}}\right)\left(\frac{1}{2}+\frac{k_{b}}{2 k_{2}}\right) e^{-i k_{4} d / s s,}
\end{gathered}
$$

where $s s=\left(1 / 2+k_{b} / 2 k_{2}\right)^{2} e^{-i k_{4} d}-\left(1 / 2-k_{b} / 2 k_{2}\right)^{2} e^{i k_{5} d}, \Delta k$ $=2 k_{1}-k_{2}$ is the phase mismatch, and $k_{3}=2 k_{1}+k_{2}, k_{4}$ $=k_{2}-k_{b}, k_{5}=k_{2}+k_{b}$. It is clear from Eqs. (8a) and (8b) that the forward and backward propagating waves, whose wave vectors are $\Delta k$ and $k_{3}$ respectively, both contribute to the transmitted and reflected SH waves. In the case of phase match, namely, $2 k_{1}=k_{2}, \Delta k=0, b=s_{2}=0$, the contribution from backward propagating wave vanishes. After $E_{t}$ and $E_{r}$ are solved, the amplitudes of homogeneous SH waves $E_{i}^{+}, E_{i}^{-}$in each unit cell of the nonlinear superlattice can be calculated according to Eqs. (5) and (7). Then the distribution of the SH field in the superlattice can be calculated directly from Eq. (2). It should be noted that nonlinear optics of multilayer thin film can also be solved by a Green's function formalism developed by Sipe and co-workers. ${ }^{19,20}$ However, as the nonlinear medium is excited by a plane wave, it is more convenient to apply the transfer-matrix approach, which is essentially based on a plane-wave expansion formalism.

The SVA approximation assumes that the amplitude of the $\mathrm{SH}$ wave is a slowly varying function, namely, $\mathrm{E}_{2}(z)$ $=F(z) e^{i k_{2} z}$, where $F(z)$ satisfies $\left|\partial^{2} / \partial z^{2} F(z)\right|$ $\ll\left|k_{2} \partial / \partial z F(z)\right|$. Then the second-order wave equation of Eq. (1) can be simplified into a simple first-order differential equation, which can be integrated out for the superlattice as

$$
\begin{aligned}
F(d) & =\int_{0}^{d} \frac{i k_{0}^{2}}{2 k_{2}} \chi(z) E^{2} e^{i \Delta k z} d z \\
& =E_{t}=\sum_{i=1}^{n} E_{i}\left(e^{i \Delta k z_{i}}-e^{i \Delta k z_{i-1}}\right),
\end{aligned}
$$

and $F(0)=0$ in the absence of SH wave input. Equation (9) means that no backward propagating wave is generated from the superlattice. Compared with Eqs. (8a) and (8b), this approximation works exactly only when the coefficients of $b$ $=s_{1}=s_{2}=0$, and $a=-1$. It requires the phase matching condition of $\Delta k=0$ and a match of refractive index for crystal and background dielectric, i.e., $n_{b}=n(2 \omega)$ and $k_{2}=k_{b}$, for example, an infinite nonlinear crystal. At other cases, the SVA approximation will result in some inaccuracies. Of course, as the dispersion is weak for many nonlinear crystals, then $\Delta k \ll k_{2} \approx 2 k_{1}$, and we have $|b| \ll|a|, \quad s_{1}=0$, and 


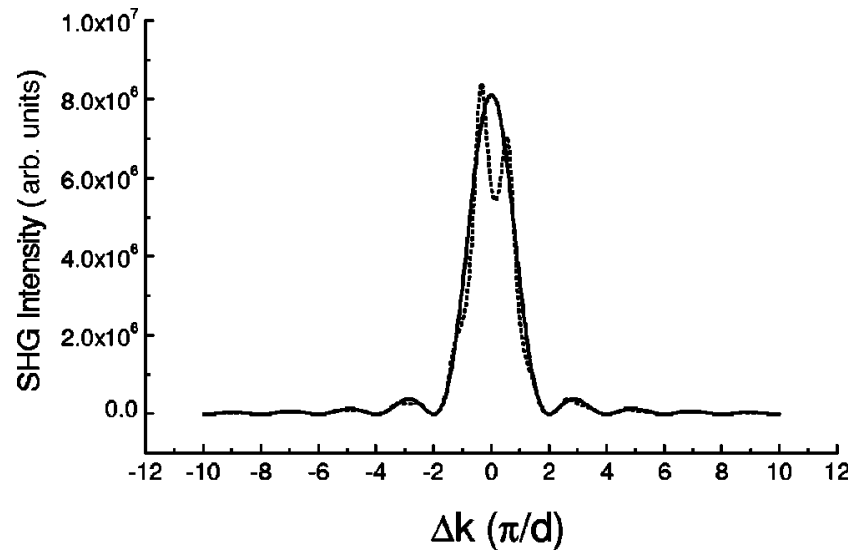

FIG. 2. Dependence of the second-harmonic generation signal on the phase mismatch for $n_{b}=n_{2}$ (solid line) and $n_{b}=1.0$ (dotted line).

$\left|s_{2}\right| \ll|a|$ at the refractive index match. In this case the contribution of the backward propagating wave to SHG can be neglected, unless the summation terms with a large wave vector of $k_{3}$ are much greater than the $\Delta k$ term.

Physically, the SVA approximation means that the energy transferred from the FW to the $\mathrm{SH}$ wave is significant only after the waves travel over a distance much longer than their wavelengths. However, in the nonlinear superlattices, the modulation of nonlinear susceptibility $\chi(z)$ may result in a rapid variation of the SH field. In addition, the multireflection effect of interfaces between unit cells as well as between the crystal and the background medium will generate nonnegligible backward propagating SH wave. Therefore, significant deviation from the conventional SVA approximation may be expected in such nonlinear superlattices.

We first consider a homogeneous nonlinear crystal, where $\chi(z)$ in Eq. (2) is a constant. Parameters of the system are taken as: Crystal length $d=100 \mu \mathrm{m}$, wavelength of FW $\lambda$ $=1.064 \mu \mathrm{m}$, the refractive indices of the FW and SHG are $n_{1}=2.1404$ and $n_{2}=2.1403$, respectively. The coherence length of the system is $\Delta l=\pi / \Delta k=2661 \mu \mathrm{m}$, far larger than the crystal length, allowing a phase match condition. It is well-known in nonlinear optics that when the SVA approximation holds, SHG reaches its maximum value at phase match $\Delta k=0$. However, the multireflection effect occurring at interfaces may compete with the phase-match effect in achieving the maximum SHG. This was verified by the numerical simulation. The dependence of the SHG signal (transmitted wave) on the phase mismatch for the homogeneous crystal with $n_{b}=n_{2}$ and $n_{b}=1.0$ is shown in Fig. 2 by solid and dotted lines, respectively. Here $n_{1}$ is fixed at 2.1404 , and $\lambda=1.064 \mu \mathrm{m}$. The maximum of SHG for $n_{b}$ $=1.0$ lies at $\Delta k=-0.34(\pi / d)$ instead at $\Delta k=0$ for $n_{b}$ $=n_{2}$. Another lower signal peak lies at $\Delta k=0.54(\pi / d)$. This difference can be attributed to the multiple reflections from the crystal-air interface, which can be well described by the interference factor $s s=\left(1 / 2+k_{b} / 2 k_{2}\right)^{2} e^{-i k_{4} d}-(1 / 2$ $\left.-k_{b} / 2 k_{2}\right)^{2} e^{i k_{5} d}$ in Eqs. (8a) and (8b). This factor is unity at $n_{b}=n_{2}\left(k_{b}=k_{2}\right)$, while it is a rapid oscillating function at $n_{b} \neq n_{2}$. Irrespective of remarkable difference for a small phase mismatch, the SHG in both cases decays rapidly as the phase mismatch increases. It should be noted that the posi-

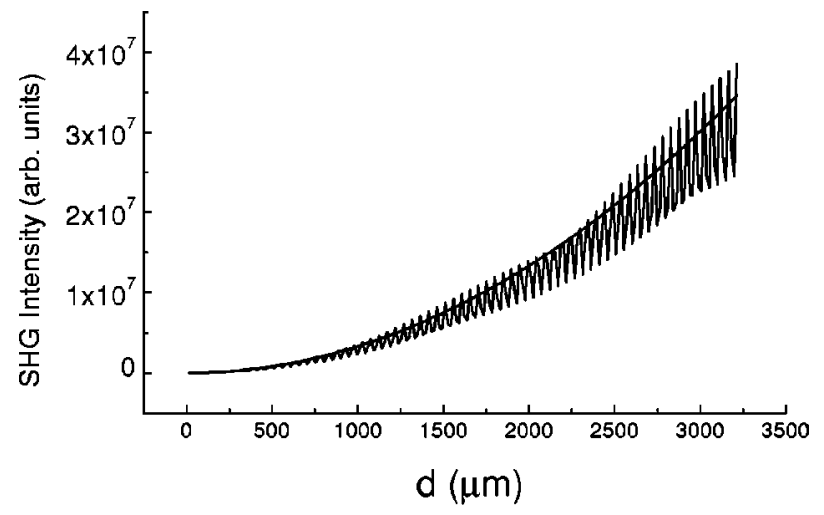

FIG. 3. Plots of second-harmonic generation signal as a function of crystal length for $n_{b}=n_{2}$ (smooth line) and $n_{b}=1.0$ (oscillating curve) in a periodic $\mathrm{TaLiO}_{3}$ superlattice with a period of $a$ $=16.07 \mu \mathrm{m}$.

tion of signal peak varies versus the crystal length, and in some cases the peak is at $\Delta k=0$.

Investigations of the SH field inside the crystal also demonstrate the violation of the SVA approximation when the reflection from the crystal interface is appreciable. The $\mathrm{SH}$ field in Eq. (2) can be rewritten as $\mathrm{E}_{2}(z)=\left(f_{r}+\mathrm{i} f_{i}\right) e^{i k_{2} z}$ $+\left(b_{r}+\mathrm{i} b_{i}\right) e^{-i k_{2} z}$, where $f_{r}, f_{i}, b_{r}$, and $b_{i}$ are the real and imaginary parts of complex amplitudes, respectively. The SVA approximation remains reasonable only when $b_{r}$ and $b_{i}$ are small compared to $f_{r}$ and $f_{i}$. It was found that at refractive index match, $f_{r}$ and $f_{i}$ are both proportional to the propagation distance, and $b_{r}$ and $b_{i}$ are both negligible, the SVA approximation works accurately. However, when the mismatch of refractive index occurs, $b_{r}$ and $b_{i}$ are comparable to $f_{r}$ and $f_{i}$, then the SVA approximation does not hold.

We now consider a realistic nonlinear material of $\mathrm{LiTaO}_{3}$, where $n_{1}=2.1404$ and $n_{2}=2.1073$ at $\lambda=1.064 \mu \mathrm{m}$, and the coherent length is $\Delta l=8.036 \mu \mathrm{m}$. The quasiphase match can be obtained in periodic or quasiperiodic superlattice with reciprocal vectors equal to the phase mismatch $\Delta k$ or its integer multiples, ${ }^{9,10,13-15}$ as according to Eqs. (8a) and (8b), only the first sum term (forward propagating wave) contributes to the SH waves. As an example, we have calculated the SH field inside periodic superlattices with a period of $a$ $=2 \Delta l=16.07 \mu \mathrm{m}$. The behavior of a SHG signal appears similar with the above homogeneous crystal. At refractive index match of $n_{b}=n_{2}=2.1073$, the backward propagating SH wave is negligible $\left[s_{1}=0\right.$ at Eqs. (8b)] due to the absence of interface multireflection. Therefore, $b_{r}=b_{i}=0$, and $f_{r}$ and $f_{i}$ increase linearly with propagation length, which results in a parabolic dependence of SHG signal on the crystal length, as shown in Fig. 3 by the smooth line. In contrast, at refractive index mismatch of $n_{b}=1.0, b_{r}$ and $b_{i}$ are comparable with $f_{r}$ and $f_{i}$. The SH field inside the superlattice oscillates rapidly, so does the SHG signal versus the crystal length, as shown in Fig. 3 by the oscillating curve. This means that the SVA approximation does not hold any more.

In addition to the interface reflection, the rapid variation of the nonlinear susceptibility $\chi(z)$ may result in the failure of the SVA approximation, since in such superlattices the backward propagating wave can overwhelm the SHG. This 
can be clearly seen in Eqs. (8a) and (8b). When $\chi(z)$ varies rapidly in a length comparable with the $\mathrm{SH}$ wavelength, the first sum term (forward propagating wave) in Eqs. (8a) and (8b) vanishes due to the rapid oscillation of the coefficient $E_{i}$, while the second sum term (backward propagating wave) dominates in the SHG. Therefore, in such short-period nonlinear superlattices, the SVA approximation fails completely. In fact, recently such a backward SHG configuration has been extensively investigated in both theoretical and experimental sides. ${ }^{16-18}$ However, the SVA approximation was still adopted in the theoretical discussions.

To verify the above assumption, we have considered a periodic superlattice with a period of $a=5 \times\left(2 \pi / k_{3}\right)$ $=0.63 \mu \mathrm{m}$ and a length of $d=125 \mu \mathrm{m}$. According to Eqs. (8a) and (8b), this superlattice can achieve a quasi-phase match for the backward propagating wave instead of the forward propagating wave. The simulation results demonstrate that the amplitude of backward wave $\left(b_{r}\right.$ and $\left.b_{i}\right)$ increases with respect to its propagation distance (along the negative $\mathrm{z}$ axis), and overwhelms that of the forward wave $\left(f_{r}\right.$ and $\left.f_{i}\right)$. Even in the absence of reflection from the crystal interface $\left(n_{b}=n_{2}\right)$, the forward $\mathrm{SH}$ wave is negligible compared with the backward wave. It is also found that when the period of the superlattice decreases, the efficiency of SHG will increase remarkably.

In the above discussions, we have assumed the approximation of negligible pump power depletion. If the conversion efficiency of SHG is high, one has to solve the nonlinear coupled wave equations satisfied with the FW and the $\mathrm{SH}$ fields. It is noted above that in the SVA approximation, the amplitude of the SH wave is a slowly-varying function, whose second derivative can be neglected. Then the secondorder coupled wave equations can be simplified into much simpler first-order coupled differential equations. ${ }^{1}$ This approximation greatly reduces the difficulty of solving the nonlinear coupled equations. So, it may be expected that many phenomena in nonlinear optics should be appraised in a more rigorous way when the SVA approximation fails. This involves the solution of nonlinear coupled second-order differential equations, a difficult task that needs further extensive investigations.

In summary, we have developed a transfer-matrix method to appraise the validity of the SVA approximation which is widely adopted in nonlinear optics. In particular, rigorous solutions of SHG from nonlinear optical superlattices show that the SVA approximation is invalid when the reflection of $\mathrm{SH}$ wave from the crystal interface is appreciable. Furthermore, when the modulation period of superlattice is comparable to the wavelength of the $\mathrm{SH}$ wave, the approximation is far from accurate even when no reflection from the crystal interface occurs. The transfer-matrix method may provide a novel way to investigate various nonlinear optical processes in superlattices in a precise way.

This work was supported by the National Natural Science Foundation of China. Z.Y.L. gratefully acknowledges financial support from Hong Kong RGC Grant No. HKUST 6112/ 98P.
${ }^{1}$ Y. R. Shen, The Principles of Nonlinear Optics (Wiley, New York, 1984).

${ }^{2}$ N. Bloembergen, Rev. Mod. Phys. 54, 685 (1982).

${ }^{3}$ P. A. Franken, A. E. Hill, C. W. Peters, and G. Weinreich, Phys. Rev. Lett. 7, 118 (1961).

${ }^{4}$ J. A. Armstrong, N. Bloembergen, J. Ducuing, and P. S. Pershan, Phys. Rev. 127, 1918 (1962).

${ }^{5}$ N. Bloembergen, Nonlinear Optics (Benjamin, New York, 1965).

${ }^{6}$ G. D. Boyd and D. A. Kleiman, J. Appl. Phys. 39, 3597 (1968).

${ }^{7}$ S. Smokeh and A. Yariv, Opt. Commun. 6, 301 (1972).

${ }^{8}$ D. Feng et al., Appl. Phys. Lett. 37, 607 (1980).

${ }^{9}$ S. N. Zhu et al., Phys. Rev. Lett. 78, 2752 (1997).

${ }^{10}$ S. N. Zhu, Y. Y. Zhu, and N. B. Ming, Science 278, 843 (1997).

${ }^{11}$ M. Pierrou, F. Laurell, H. Karlsson, T. Kellner, C. Czeranowsky, and G. Huber, Opt. Lett. 24, 205 (1999).

${ }^{12}$ G. M. Gibson, M. Ebrahimazadeh, M. J. Padgett, and M. H. Dunn, Opt. Lett. 24, 397 (1999).

${ }^{13}$ G. W. Ross et al., Opt. Lett. 23, 171 (1998).

${ }^{14}$ N. Ramanujam and J. J. Burke, IEEE J. Quantum Electron. 33, 152 (1997).

${ }^{15}$ M. M. Fejer, G. A. Magel, D. H. Junt, and R. L. Byer, IEEE J. Quantum Electron. 28, 2631 (1992).

${ }^{16}$ P. St. J. Russell, IEEE J. Quantum Electron. 27, 830 (1991).

${ }^{17}$ Y. J. Ding and J. B. Khurgin, Opt. Lett. 21, 1445 (1996).

${ }^{18}$ X. Gu, R. Y. Korotkov, Y. J. Ding, J. U. Kang, and J. B. Khurgin, J. Opt. Soc. Am. B 15, 1561 (1998).

${ }^{19}$ J. E. Sipe, J. Opt. Soc. Am. B 4, 481 (1987).

${ }^{20}$ V. Mizrahi and J. E. Sipe, J. Opt. Soc. Am. B 5, 660 (1988). 